University of Nebraska - Lincoln

DigitalCommons@University of Nebraska - Lincoln

CSE Conference and Workshop Papers

Computer Science and Engineering, Department

2005

\title{
Discontinuous Waveband Switching in WDM Optical Networks
}

Bhavana Lekkala

Byrav Ramamurthy

University of Nebraska-Lincoln, bramamurthy2@unl.edu

Follow this and additional works at: https://digitalcommons.unl.edu/cseconfwork

Part of the Computer Sciences Commons

Lekkala, Bhavana and Ramamurthy, Byrav, "Discontinuous Waveband Switching in WDM Optical Networks" (2005). CSE Conference and Workshop Papers. 76.

https://digitalcommons.unl.edu/cseconfwork/76

This Article is brought to you for free and open access by the Computer Science and Engineering, Department of at DigitalCommons@University of Nebraska - Lincoln. It has been accepted for inclusion in CSE Conference and Workshop Papers by an authorized administrator of DigitalCommons@University of Nebraska - Lincoln. 


\title{
Discontinuous Waveband Switching in WDM Optical Networks
}

\author{
Bhavana Lekkala and Byrav Ramamurthy \\ Department of Computer Science and Engineering \\ 256 Avery Hall, University of Nebraska-Lincoln \\ Phone: 402-472-7791, Fax: 402-472-7767. \\ Email: \{bhavanal, byrav\}@cse.unl.edu
}

\begin{abstract}
Routing techniques used in wavelength routed optical networks (WRN) do not give an efficient solution with Waveband routed optical networks (WBN) as the objective of routing in WRN is to reduce the blocking probability and that in WBN is to reduce the number of switching ports. Routing in WBN can be divided two parts, finding the route and grouping the wavelength assigned into that route with some existing wavelengths/wavebands. In this paper, we propose a heuristic for waveband routing, which uses a new grouping strategy called discontinuous waveband grouping to group the wavelengths into a waveband. The main objective of our algorithm is to decrease the total number of ports required and reduce the blocking probability of the network. The performance of the heuristic is analyzed using simulation on a WBN with non-uniform wavebands.
\end{abstract}

Index Terms-Optical fiber switches, optical interconnection, routing, waveband switching.

\section{INTRODUCTION}

Wavelength-division multiplexing (WDM) divides the optical transmission spectrum into non-overlapping wavelengths (frequencies). Each wavelength is used as a communication channel and can be operated asynchronously at different speeds. The channels are then optically multiplexed such that multiple channels travel down the fiber simultaneously and with negligible interference.

As the traffic demand increases, the complexity of switching is also increasing. Switching is thus becoming a cost-performance bottleneck. The cost and scalability concerns of switching nodes prompted the creation of multiple switching granularities, such as wavelengths and wavebands $[1,2]$. A waveband consists of several wavelengths and needs only two ports of an optical switch in a node. A waveband can be switched optically as a single unit, thus reducing the number of optical-electrical-optical ports that are required for processing individual wavelengths.

Routing in waveband routed optical networks (WBN) is different from the routing in wavelength routed optical networks (WRN). Each of them has different objectives so WRN's routing techniques cannot be applied effectively to WBN. More specifically, in networks with ordinary optical crossconnects $(\mathrm{OXC})$, the routing and wavelength assignment

This work was supported in part by the U. S. National Science Foundation grants (ANI-0074121 and EPS-0091900) and the UNL Program of Excellence PRISM Priority Initiative.
(RWA) problem is to find the best route and assign a wavelength to it. So the objective of RWA is to minimize the blocking probability or maximize the number of connections established. This problem is proved to be NP-complete. However, the main objective of routing in WBN is to minimize the number of switching ports and thus reduce the cost of switching devices.

Routing in WBN can be divided two parts, finding the route and grouping the wavelength assigned to that route with some existing wavebands. The previous work [1-3] studied various grouping techniques but used the traditional shortest path algorithm to find the best route. Shortest path routing in WBN does not always give an efficient solution to the problem. Different grouping strategies may improve the performance but may not give the best solution.

In this paper we propose a new routing algorithm for WBN and a new waveband grouping strategy whose objective is to minimize the number of switching ports and minimize the blocking probability. It also considers RWA as an important part of routing. The rest of the paper is organized as follows. In Section II we discuss the existing routing and grouping techniques proposed in earlier studies. In Section III, we present a new algorithm and in Section VI we discuss how the performance of the new algorithm is measured using simulation. In Section $\mathrm{V}$ we discuss some of the advantages and disadvantages of the new algorithm. Section IV proposes future extensions that can be made to improve the algorithm.

\section{NODE ARCHITECTURE AND GROUPING STRATEGY IN WAVEBAND SWITCHING}

To reduce the complexity of switching devices multigranularity is proposed in [4-5]. The main idea behind multigranularity in optical networks is that the number of nodes in the core network is limited, and if many wavelengths are required to carry all the traffic, some of them will start from the same source node and go to the same destination nodes, and even more wavelengths will follow the same sub-paths inside the network. Thus it is useless to switch them separately through some optical nodes; they can be switched in groups called wavebands.

The traditional OXCs are only capable of switching wavelengths and so in order to switch waveband as a single unit the OXCs should be modified. Several different architectures for the new $\mathrm{OXC}$ or multi-granularity $\mathrm{OXC}$ (MOXC) have been proposed in [4,7]. Previous studies [1-3, 
5-6] have improved the MGOXC architecture based on their algorithms and requirements.

Accordingly, several grouping strategies are proposed in [1] to aggregate the wavelengths into wavebands. To group wavelengths the following grouping strategies are used in [1].

1. End-to-End grouping: If there are $m$ wavelengths or more for the traffic from the same source node to the same destination node, one can group $m$ wavelengths into one band to route this band instead of the $m$ separate wavelengths through the network.

2. Intermediate grouping shown in Fig.1:

a. Same source different destinations: We can also group the wavelengths starting from the same source but which are going to different destinations but share a common sub-path.

b. Different sources same destination: Similar to the above grouping, we can also group the wavelengths that have different sources but are going to the same destination and have a common sub-path.

B efore grouping
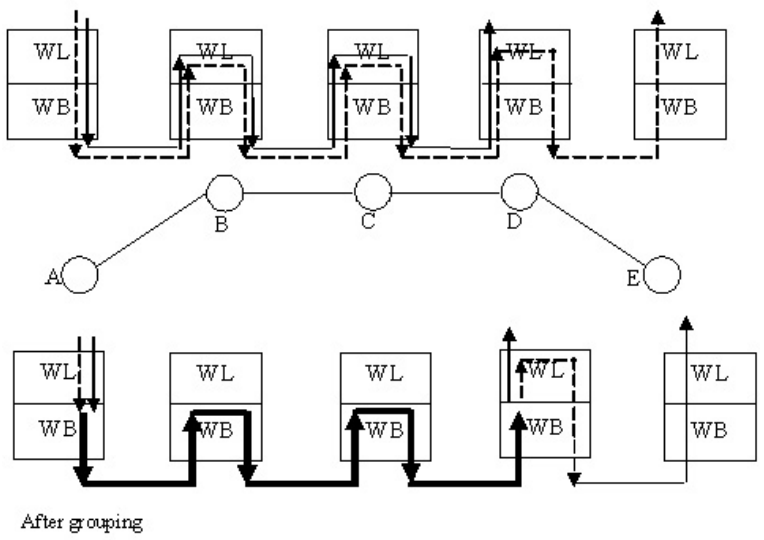

WL: Wavelength Layer WB: Waveband Layer

Fig. 1. Intermediate Waveband Grouping

For networks with few nodes, intermediate grouping can bring a reduction of parts by a factor 2 than end-to-end grouping. For networks with more nodes, end-to-end grouping proves to be even less efficient than intermediate grouping [1]. A study [2] simulated intermediate grouping with the different source and different destination scheme and proved that it performs better than the other schemes.

All the papers till now assumed that the number of wavelengths required to form a waveband is constant throughout the network but [5] proposed non-uniform wavebands (wavebands containing different number of wavelengths). Their simulations showed significant cost reduction in the case of both ring and mesh networks by using non-uniform wavebands rather than uniform wavebands.

We believe that traditional RWA algorithms cannot be directly applied to waveband routing. In our paper we propose a wavelength grouping strategy and a waveband routing heuristic for non-uniform waveband routed optical networks. A set of wavelengths form a waveband and this number is assumed to be constant for the entire network in most of the studies. Having non-uniform wavebands will improve the performance of waveband switching according to [5]. In our study we assume non-uniform wavebands.

Based on the intermediate waveband switching, we propose another grouping strategy to efficiently group the wavelengths into wavebands, called discontinuous waveband grouping (DCBG).

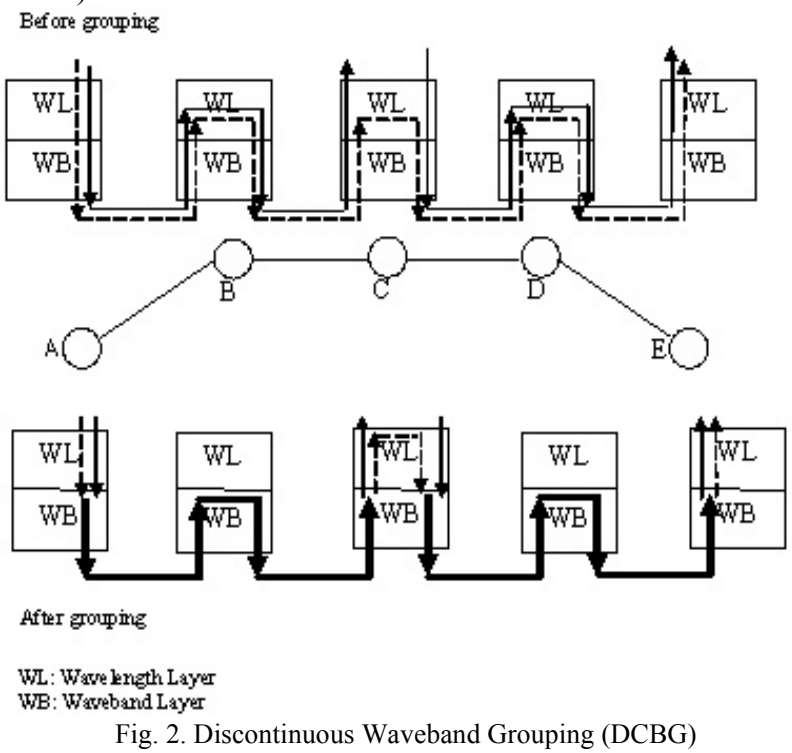

DCBG is an extension of Intermediate grouping with different source and different destination. A wavelength can be a part of multiple wavebands in its route. It does not stop grouping until the destination is reached. The detailed explanation of DCBG is given in the next section. In DCBG a wavelength path can be a part of multiple wavebands in its entire route. As shown in Fig. 2, there is a wavelength (w1) that is going from node $\mathrm{A}$ to node $\mathrm{E}$ and there are two other wavelengths (w2, w3) that are going from node A to node $\mathrm{C}$ and from node $\mathrm{C}$ to node $\mathrm{E}$. So DCBG forms a waveband with $\mathrm{w} 1$ and $\mathrm{w} 2$ in the route $\mathrm{A} \rightarrow \mathrm{B} \rightarrow \mathrm{C}$ and forms another waveband with $w 1$ and $w 3$ in the route $C \rightarrow D \rightarrow E$. This way the wavelength path $\mathrm{w} 1$ is a part of two wavebands in its route. DCBG tries to make a wavelength path as a part of either an existing waveband or by forming a new waveband there by minimizing the switching of the single wavelength paths.

DCBG does not cause any overhead for routing because it selects the wavebands that starts and terminates in the route. Wavebands need not be dropped just to merge other wavelengths. To be more precise while trying to find the best waveband sequence for a route we check whether the current waveband spans either the entire route or the subpath of the route. If the waveband spans the entire route then it is same source same destination waveband, if it does not then it should be a subpath which means that the waveband starts and terminates in the route and need not be dropped to merge the wavelengths of the current route into that waveband.

\section{DISCONTINUOUS WAVEBAND GROUPING ALGORITHM}

With non-uniform wavebands and discontinuous waveband grouping strategy we propose a heuristic algorithm for 
waveband routed optical networks. This is different from all the previous work in the following aspects:

1. We propose a new grouping strategy, discontinuous waveband grouping, which is proved to achieve more savings in terms of port count than any other grouping strategy.

2. All previous works except [5] assumed that the number of wavelengths in a waveband is constant throughout the network. [5] proposed non-uniform wavebands but it did not analyze the various grouping strategies. We have applied non-uniform wavebands to the discontinuous waveband grouping strategy.

3. Another important assumption is the number of shortest paths considered while trying to group the wavelengths. [1] considered $k$-paths while others considered only the shortest path. We have taken $k$ paths to find the best waveband route for a given request.

Using the Yen's algorithm and DCBG we propose a new heuristic for waveband switching. The heuristic objective is to minimize wbdiff, where wbdiff is the difference between the length of the route and the length of the waveband path with which the current request in that route can be merged. A route with minimum(wbdiff) is selected so as to maximize the waveband formation and minimize the wavelength switching. This will ensure that we use less number of wavelength switching ports, which in turn reduces the overall switching ports used for the same traffic.

The heuristic starts with finding $k$ shortest paths between all pairs of nodes in the network. Traffic is given as a matrix and is satisfied in two ways: Heavy traffic first, Light traffic first. The results for both strategies are compared in the next section.

For each traffic request the heuristic will find the $\mathrm{k}$ shortest paths through which the traffic can be routed and will find the existing wavebands in those paths. It computes wbdiff for each shortest path with the current status of the wavebands in those routes. A path with minimum wbdiff is selected to be a best route for the current traffic request. If a path's wbdiff is zero it means that there is a waveband that spans the entire path and has room for the current traffic request. The current traffic request is merged with the best waveband sequence.

The heuristic also tries to form a waveband if the traffic requested is greater than the minimum number of wavelengths that can be merged into a waveband. If the network does not have sufficient wavelengths in one path then the traffic is divided between $k$ shortest paths.

Partial traffic can also be satisfied depending on the current network resources so this heuristic also reduces the blocking probability as it tries to satisfy as much traffic as possible.

The detailed description of the heuristic is presented below:

Step 1. For a given traffic request from source $(s r c)$ to destination (dst) we compute the $k$-shortest paths.

Step 2. For all $k$-shortest paths check to see if there are any wavebands that span the entire route and has room for the current traffic request. If such a waveband exists then merge the current traffic request into that waveband.
Step 3. If there is no such waveband then for each shortest path find the best waveband sequence, using DCBG algorithm, with which the current wavelength path can be merged. Then compute the length of that waveband sequence.

Step 4. Find the difference (wbdiff) between the length of the wavelength path and the length of the waveband sequence for each shortest path.

Step 5. Select the shortest path with minimum wbdiff. This will make sure that we don not use more network resources even though we don not select the shortest route.

Step 6. The route with Minimum (wbdiff) is selected and the current traffic request takes the corresponding route.

Step 7. If there are no wavebands with which the current traffic request can be merged then check if the traffic requested is greater than the minimum number of wavelengths that can form a waveband.

Step 8. If the traffic requested is sufficient to form a waveband then find the shortest path which has room for the requested traffic and form a new waveband in that path.

Step 9. If the traffic requested is not sufficient to form a new waveband then find the best route using traditional wavelength routing algorithms and switch the traffic as a wavelength.

Step 10. After all traffic requests are satisfied compute the total number of ports used.

Step 11. If all the k-paths don not have enough number of wavelengths to satisfy the current traffic request then satisfy the maximum amount of traffic.

Step 12. Keep track of wavebands and wavelengths as you route the new traffic.

Step 13. Count the number of waveband ports used and the number of wavelength ports used after all the traffic requests are satisfied.

Step 14. The traffic that cannot be satisfied is considered to be blocked. It can be an entire traffic request or partial traffic.

Step 15. Number of blocked calls / Total number of calls give the blocking probability .

Step 16. The blocking probability for this heuristic is shown in the next section.

The total number of ports used using waveband switching and regular wavelength switching is compared in the results section. Different traffic acceptance patterns, heavy traffic demand first and light traffic demand first, are compared against pure wavelength switching and the results are presented in the next section.

\section{EXPERIMENTAL RESULTS}

We conduct the simulation on the 23-nodes EON network and we assume the network to be homogeneous i.e., all nodes in the network have the capability of switching wavebands. Traffic is static and is dynamically generated. The traffic matrix is given as input to the simulation. Traffic request need not necessarily be a multiple of the waveband granularity. Traffic granularity is in wavelengths. The waveband size 
varies across the network between a minimum value and a maximum value. Input parameters determine the minimum number of wavelengths per waveband and maximum number of wavelengths per waveband.

Our proposed strategy called discontinuous grouping is used to group wavelengths into a waveband. $K$-shortest paths are considered to find the best route. One of the $k$-shortest paths is selected based on the availability of wavebands in that path. Selecting the path with minimum (wbdiff) will make sure that we don not consume more network resources even though we do not select the shortest route (more detailed explanation is given in the previous section). The traffic is satisfied in two orders: Heavy traffic demand first, Light traffic demand first. Results for both orders in shown in this section. Partial traffic request can also be satisfied. If the number of wavelengths requested is not available in all the $k$ paths then the maximum number available is satisfied. If the traffic is not satisfied then it is considered to be blocked. The output of the simulation is: Number of ports saved, Number of waveband ports used, Number of wavelength ports used, and number of calls blocked.

Fig. 3 and Fig. 4 show the results, where the number of wavelengths per fiber $=8$; minimum number of wavelengths that can form a waveband $=2$; maximum number of wavelengths in a waveband $=8$; traffic acceptance pattern: Maximum Traffic First. The figures show a huge difference in the number of ports used and almost no change in the number of blocked calls.

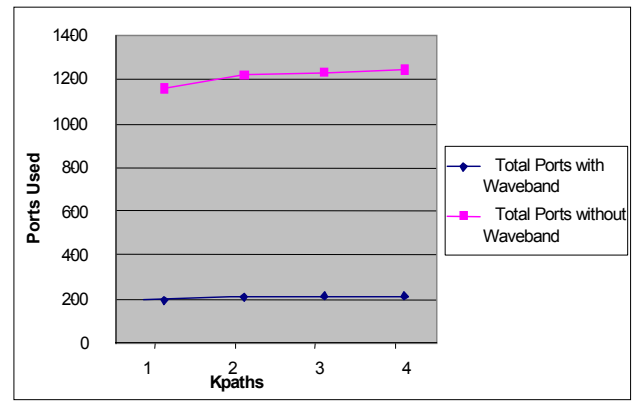

Fig. 3. Number of ports used in DCBG under the maximum traffic first policy when the number of wavelengths is 8 , the maximum waveband granularity gmax is 8 , and the minimum waveband granularity gmin is 2 .

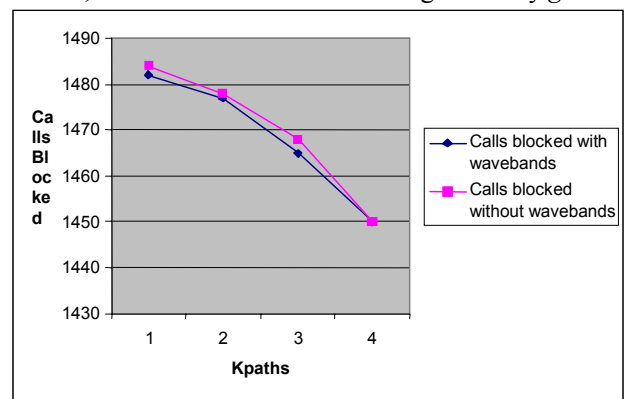

Fig. 4. Number of calls blocked in DCBG under the maximum traffic first policy when the number of wavelengths is 8 , the maximum waveband granularity gmax is 8 , and the minimum waveband granularity gmin is 2 .

Fig. 4 and Fig. 5 show the number of ports used and the number of calls blocked for the following parameters: number of wavelengths per fiber $=8$; minimum number of wavelengths that can form a waveband $=2$; maximum number of wavelengths in a waveband $=8$; traffic acceptance pattern: Minimum Traffic First. Both figures show a huge difference in the number of ports used and almost no change in the number of blocked calls.

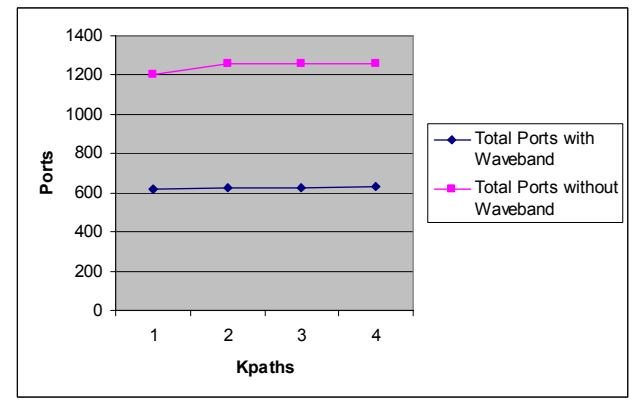

Fig. 5. Number of ports used in DCBG under the maximum traffic first policy when the number of wavelengths is 8 , the maximum waveband granularity gmax is 8 , and the minimum waveband granularity gmin is 2 .

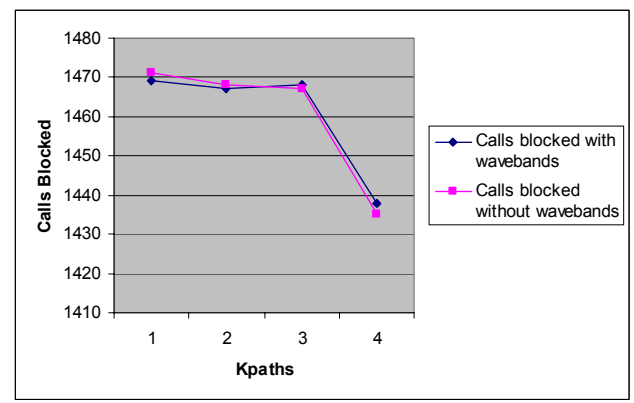

Fig. 6. Number of calls blocked in DCBG under the minimum traffic first policy when the number of wavelengths is 8 , the maximum waveband granularity gmax is 8 , and the minimum waveband granularity gmin is 2 .

We observed an interesting behavior with Maximum Traffic First pattern and Minimum Traffic First pattern shown in Fig. 7. When Maximum Traffic First pattern is used the total number of ports used is less than the total number of ports used with Minimum Traffic first pattern. The following graph (Fig. 7) shows the difference.

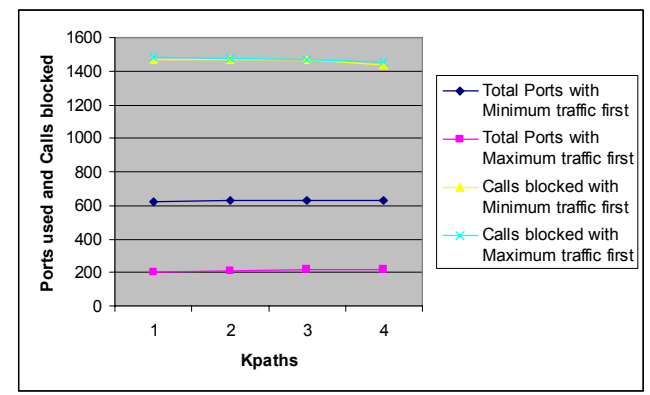

Fig. 7. Comparison of the number of ports used and calls blocked in DCBG under the maximum traffic first policy and the minimum traffic first policy.

Fig. 8 and Fig. 9 show the number of ports used and the number of calls blocked for the following parameters: Number of wavelengths per fiber $=16$; Minimum number of wavelengths that can form a waveband $=2$; Maximum number of wavelengths in a waveband $=8$; Traffic acceptance pattern: Maximum Traffic First. Both figures show a huge difference in the number of ports used and almost no change in the number of blocked calls.

Fig. 10 and Fig. 11 show the number of ports used and the number of calls blocked for the following parameters: Number of wavelengths per fiber $=16$; Minimum number of 
wavelengths that can form a waveband $=2$; Maximum number of wavelengths in a waveband $=8$; Traffic acceptance pattern: Minimum Traffic First. The graphs show a huge difference in the number of ports used and almost no change in the number of blocked calls.

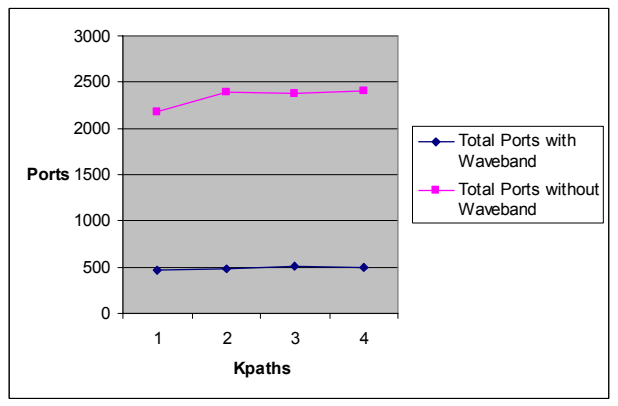

Fig. 8. Number of ports used in DCBG under the maximum traffic first policy when the number of wavelengths is 16 , the maximum waveband granularity gmax is 8 , and the minimum waveband granularity gmin is 2 .

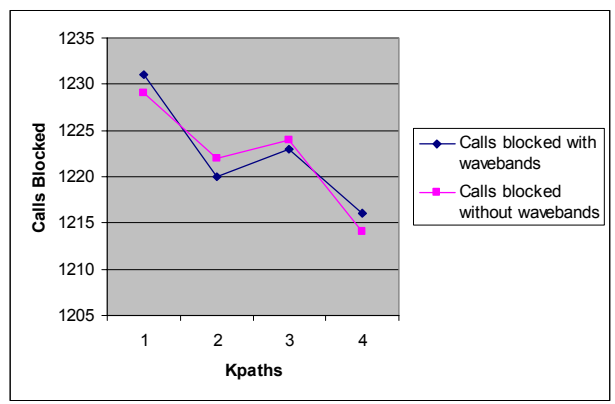

Fig. 9. Number of calls blocked in DCBG under the maximum traffic first policy when the number of wavelengths is 16 , the maximum waveband granularity gmax is 8 , and the minimum waveband granularity gmin is 2 .

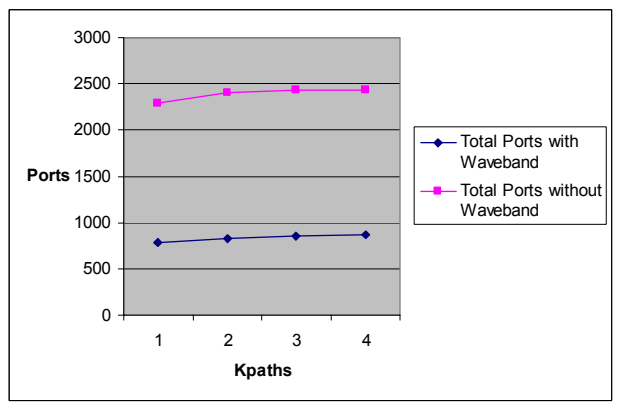

Fig. 10. Number of ports used in DCBG under the minimum traffic first policy when the number of wavelengths is 16 , the maximum waveband granularity gmax is 8 , and the minimum waveband granularity gmin is 2 .

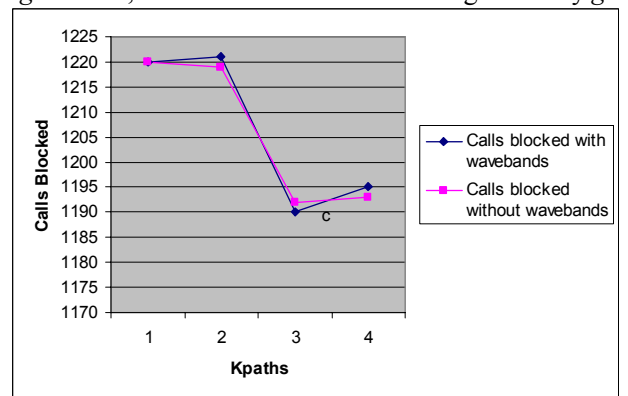

Fig. 11. Number of calls blocked in DCBG under the minimum traffic first policy when the number of wavelengths is 16 , the maximum waveband granularity gmax is 8 , and the minimum waveband granularity gmin is 2 .

\section{CONCLUSION AND FUTURE WORK}

In this paper we proposed a new wavelength grouping strategy and proposed a heuristic for waveband routing. The port savings using our heuristic is shown in our results. Our heuristic is the first one to consider K-shortest paths, nonuniform wavebands and discontinuous grouping strategy for routing wavebands.

Future extensions of this work will be to implement this algorithm for heterogeneous networks where only some nodes in the network have the capability of switching wavebands. This algorithm can also be adapted to a dynamic traffic scenario where calls stay in the network for a certain period.

\section{ACKNOWLEDGMENT}

The authors thank Ms. Mengke Li, a a Ph.D. candidate in the Department of Computer Science and Engineering at the University of Nebraska-Lincoln, for her contributions towards the preparation of this manuscript.

\section{REFERENCES}

[1] L. Noirie, M. Vigoureux, and E. Dotaro, "Impact of intermediate traffic grouping on the dimensioning of multi-granularity optical networks," Proc. of Optical Fiber Communication [OFC'2001] (Anaheim, CA, USA, March 2001), pp. TuG3/1-3.

[2] R. Lingampalli and P. Vengalam, "Effect of wavelength and waveband grooming on an all-optical networks with single layer photonic switching", Proc. of Optical Fiber Communication [OFC'2002] (Anaheim, CA, USA, March 2002). ThP4/1-3.

[3] Y. Suemura, I. Nishioka, Y. Maeno, S. Araki, R. Izmailov, and S. Ganguly, "Hierarchical routing in layered ring and mesh optical networks," Proc. IEEE ICC'2002 (New York, NY, USA, April-May 2002), vol. 5, pp. 2727-2733.

[4] K. Harada, K. Shimizu, T.Kudou and T.Ozeki, "Hierarchical Optical Path Cross-Connect Systems for Large Scale WDM Networks," Proc. of Optical Fiber Communication [OFC'2001], poster WM55.

[5] Ernesto Ciaramella, "Introducing Wavelength Granularity to Reduce the Complexity of Optical Cross Connects," IEEE Photonics Technology Letters, vol. 12, no. 6, June 2000.

[6] R. Izmailov, S. Ganguly, V. Kleptsyn, and A. C. Varsou, "Non-uniform waveband hierarchy in hybrid optical networks," Proc. IEEE INFOCOM'2003 (San Francisco, CA, USA, April 2003), vol. 2, pp. 1344-1354.

[7] P.-H. Ho, H. T. Mouftah, and J.Wu, "A novel design of optical crossconnects with multi-granularity provisioning support for the nextgeneration internet," Proc. IEEE ICC2003 (Anchorage, Alaska,USA, May 2003), vol. 1, pp. 582-587.

[8] X. Cao, V. Anand, Y. Xiong, and C. Qiao, "Performance evaluation of wavelength band switching in multi-fiber all-Optical networks," Proc. IEEE INFOCOM'2003 (San Francisco, CA, USA, April 2003), vol. 3, pp. 2251-2261. 